\title{
APPLICATIONS OF THE UNIVERSAL JOINT TASK LIST TO JOINT EXERCISE RESULTS
}

\author{
Sam H. Parry \\ Michael C. McAneny \\ Richard J. Dromerhauser \\ Department of Operations Research \\ Naval Postgraduate School \\ Monterey, CA 93943, USA
}

\begin{abstract}
The foundation of readiness is training. The Chairman, Joint Chiefs of Staff (CJCS) Joint Training Program institutes methods for identifying training requirements through review of the Commander-in-Chief's (CINC) missions and the compilation of Joint Mission Essential Task Lists. The Universal Joint Task List comprehensively outlines these joint essential tasks, providing a summary of CINC missions, joint tasks, and corresponding supporting and enabling tasks. Computer aided exercises (CAXs) are tools available for monitoring and training a staff in these tasks. CAXs are an essential part of staff training, although one of their major weaknesses is their inability to accurately measure the level of training received by the players. This paper develops an exercise analysis methodology for evaluating critical event causal audit trails. Specific objectives are to determine quantifiable measures of effectiveness designed to work with data manipulated by computer simulations and to test MOEs using the Joint Theater Level Simulation (JTLS). This includes the development of post-exercise analysis techniques for wargame data. This paper provides a methodology for extracting appropriate data from a CAX to develop causal audit trails for critical events. The results of a $\mathrm{CAX}$ will then be more available for trend analysis and feedback.
\end{abstract}

\section{INTRODUCTION}

In peacetime, military professionals must acquire the skills and develop the confidence and initiative necessary to conduct joint and combined operations. While professional schools are fundamentally important, the military is a hands-on profession and most learning at all levels is accomplished while participating in unit training and operations. Hence, realistic, demanding and objectively measured training and exercises are essential. The Joint Training Program defined within the Chairman Joint Chiefs of Staff (CICS) Memorandum of Policy 26 (MOP 26) establishes a program for carrying out the joint training responsibilities of the CJCS, the Joint Commanders-inChief (CINCs), and the CINCs' component staffs. MOP 26 institutes a method for identifying training requirements through the review of the CINC's mission and the compilation of essential tasks required to accomplish that mission. Each compiled task list is called the CINC's Joint Mission Essential Task List (JMETL).

A CINC's JMETL is intended to provide the basis for all joint training. A JMETL consists of those tasks deemed essential for accomplishment of operational plans, predicated on the missions assigned and forces apportioned to the CINC, U.S. alliances or treaties, or regional initiatives. A JMETL includes Joint Mission Essential Tasks, supporting tasks considered essential for accomplishment of the Joint Mission Essential Tasks, and enabling tasks.

The Universal Joint Task List (UJTL), a supplement to the Joint Training Manual (MCM 7192), outlines a comprehensive list of joint essential tasks (JCS, 1996) and provides:

- A summary listing of CINC Missions.

- A list of Joint Tasks, the corresponding Supporting Tasks, and their Enabling Tasks.

- A detailed dictionary of the Joint Tasks, Supporting Tasks, and the Enabling Tasks, describing each task in detail.

For consistency and comparability purposes, each CINC is required to develop a JMETL based on the missions and essential tasks outlined in the Universal Joint Task List document. The CINCs are responsible for identifying their major missions from the summary listing; mapping the major missions to the joint tasks; 
determining the joint tasks which are most crucial to their missions and which occur most frequently; and identifying which tasks are in the greatest need of training.

How does a CINC go about identifying which tasks are "in the greatest need of training?" In addition to subjective post-exercise commentary, a complementary, objective MOE is needed to quantitatively evaluate staff performance in all potential Joint Mission Essential Tasks, but how is this efficiently done? One of the primary training tools available to a CINC for training and evaluating his staff in this regard is an exercise supported by a computer simulation model. This is commonly referred to as a Computer Aided Exercise (CAX). The primary role of the computer simulation is to present a decision environment within which the exercised staff can be presented with realistic situations.

Although CAXs have proved to be an essential training tool for a CINC and his staff, until recently there have been few methodologies available to quantitatively evaluate the results of the CAX. The research efforts of Combs (1995), Towery (1995), Brown (1996), Mustin (1996), Cwick (1996), Sullivan (1996), Thurman (1996), and Gordon (1996) developed individual methodologies and measures of effectiveness (MOEs) to quantify and evaluate the performance of a CINC's staff in separate and distinct mission areas. These theses covered the topics of logistics support, intelligence functions, operational maneuver, carrier battlegroup anti-air warfare, amphibious logistics, mobilization planning, force protection, and operational firepower, respectively. Given these tools, a CINC can begin to identify reasons why an exercise produced a given outcome in a given mission area. These methodologies assist in locating critical events in a scenario that significantly contribute to its outcome. After this analysis, there still exists the problem of formulating comprehensive MOEs to evaluate the staff's overall performance in all mission areas.

Simply stated, the problem is that currently there is no comprehensive tool for a CINC to look at an overall CAX and its results, accurately pinpoint critical events in any or all mission areas, and then determine why these critical events occurred. This paper develops a methodology and presents results from the Joint Theater Level Simulation (JTLS).

\section{METHODOLOGY}

Critical events are those events of a catastrophic nature that singularly or in concert with a limited number of like events could cause operational and/or strategic level consequences. Some examples of events of this nature might be the loss of a tank battalion, the destruction of a forward deployed ground unit or the completion of a successful reconnaissance mission.

Given the stochastic nature of a CAX, it is possible for critical events to occur at any point in the scenario. Because a CAX is a training tool for the CINC, the question of why the critical event occurred is of great importance. The vehicle for ascertaining the answer is the audit trail. Audit trails are created by careful examination and manipulation of the simulation's postprocessor output. The goal is to use the output to trace backward from the occurrence of a critical event in an attempt to discover the causal relationships.

Currently, the only capabilities investigators have in developing the audit trail are quantitative items such as the exact time, place and strength of a unit where the critical event occurred. If all logically required assets are present and functioning at the time of the critical event, then it might be said that the event occurred due to the stochastic nature of the model. This is the realism imparted by stochastic models. For example, a Patriot defense system may be on station; however, scuds may still penetrate the air defense envelope. Any enemy threat, even though planned for, still has a positive probability of defeating the planned defense, regardless of the level of preparation. This type of critical event is well explained through audit trail analysis. The situation gets more difficult to evaluate when pieces seem to be missing from the puzzle. If, for instance, a major supply depot is destroyed by undetected enemy air forces and it is subsequently discovered, via the audit trail, that the depot was virtually unprotected, the CINC will want to know why.

The nature of combat is such that seemingly trivial events can eventually have a large impact on battle outcomes. Compounding these events leads to a cascading effect that may, in turn, become a critical event. The nature of current audit trail analysis makes it nearly impossible to answer the question of why various events took place; this process will confirm only that they did take place. In the context of the supply depot example, the analyst can discover that an air defense unit was not located close enough to the supply depot. The question of why the unit was not close enough to provide sufficient defense still remains.

In order to standardize the process of tracing a critical event's audit trail, one must have a consistent methodology that is applicable for any type of critical event. One method that will achieve these goals is a checklist of all reasonable scenario parameters that could affect an event. One should be able to reconstruct the ground, air and/or naval situation at any historical time during a training event. By using the following checklist of general questions in the same manner for every possible critical event, the causal relationships 
gradually may become more evident, allowing for further analysis.

- Time and location: When did the critical event occur, and what were the locations of all involved units and/or targets?

- Force strength: What are the force strengths of the participating units in the critical event? Are any reasonably too low? Do any of the participating units have key combat systems casualties?

- Environmental conditions: Did weather, visibility conditions, or terrain hamper or overly assist any participating units in accomplishing their given missions?

- Command and control issues: Were units on both sides able to communicate? Were any participating units given multiple tasking or possibly confusing orders?

- Logistics: Were there any supply shortfalls? Were they due to a lack of or a misallocation of assets?

- Intelligence: Were there any intelligence shortfalls? Were they due to a lack of or a misallocation of assets?

- Subjective issues: Did this critical event happen due to unexplained miscalculations or an error in a commander's judgment, or due to chance?

Once these questions are posed and the relevant questions answered, the analyst can then, if necessary, move to the next step of generating a set of critical event specific queries.

\section{JTLS SCENARIO}

The Joint Theater Level Simulation is an interactive, multi-sided, joint (air, land, sea, and special operations) and combined (coalition warfare) constructive simulation model which is used as both a robust tool to analyze theater level operations plans and as a vehicle to support training exercises and seminar wargames. JTLS strives to model conflict at the operational level with tactical fidelity. Additionally, JTLS maintains documentation of how functional areas interact throughout the game.

JTLS uses SIMSCRIPT to support the need for a discrete time simulation. The advantage of the discrete time simulation is the ability to model activities that have been identified as critical events. The key processes of theater level, air land battle are most easily visualized as a collection of discrete (key) events. These critical events may significantly change the state of the systems.
Rolands and Associates Corporation, the developer of JTLS, has created several routines which continually update ASCII output files with critical data during the conduct of a JTLS exercise. These files have been developed in conjunction with the UJTL assessment effort and provide a variety of data describing engagement results, resupply, and a number of other characteristics. The JTLS JMET output files comprise the input to the database for subsequent data retrieval. JTLS version 2.1 employs the ORACLE Relational Database Management System (RDBMS).

The selected JTLS scenario is set in the Southwest Asia theater of operations. The conditions exhibited were of an enemy seizure of the strategic initiative, sufficiently degrading the ability to build combat power quickly in theater. The scenario resulted in long distances being covered to bring forces in contact with the enemy, demonstrating the difficulty in generating combat power and establishing a temporal advantage. This scenario is a likely one for several reasons. These reasons could relate to the occupation of friendly assets in another region or the ability of an aggressor nation to recognize the need to seize the initiative in the overall strategic situation.

The Iraqi forces in this scenario have attacked across the border to Hafir-al-Batin in north central Saudi Arabia and to the Kuwaiti border along the coast. The immediate objective was to seize the Trans-Arab pipeline and control the flow of oil in northern Saudi Arabia.

The deployment sequence was formulated to allow for one brigade each from the 101st Airborne Division and 24th Mechanized Infantry Division to arrive without difficulty at a port city near the city of Dhahran. Because the database already contained United Kingdom forces in the region, they were used to support Saudi Arabian forces in the vicinity of King Khalid Military City (KKMC). Finally, the Iraqi advance along the coast stopped short of crossing into Saudi Arabia, allowing the deployment of two Marine Expeditionary Units into the area of Al-Khafji. Under the conditions of Iraqi strategic initiative, forces attacked well in advance of any Allied presence in the region. Iraqi forces secured the operational initiative by conducting preemptive air strikes on deploying United States forces. Forces from the 2nd Brigade of the 24th Mechanized Infantry Division (2/24th Mech) were given the mission to move west and support the defense of KKMC. The Iraqi air and ground efforts were designed to impede that movement. 


\section{CAUSAL AUDIT TRAIL ANALYSIS}

\subsection{Subjectivity in Analysis}

In theory, the causal audit trail process can trace the causal factors from any critical event down to the most minute detail of combat. In reality, the process' limiting factors are a lack of significant quantitative data and the subjective battlefield decision. The causal audit trail is graphically presented as a version of a "decision tree." This tree attempts to delineate all the possible causes of a critical event, enabling the analyst to follow the path that provides the most likely cause, based on quantitative data. The branches of the tree end when the data required to determine the cause of a course of action is unreasonably large, impossible to retrieve, or when the analyst is faced with finding the cause of a commander's subjective decision.

The concept of being faced with a "subjective dead end" while performing wargaming analysis is further documented by Coleman Research Corporation's (1997) efforts to quantitatively evaluate the entire UJTL. CRC concluded that of the 5199 UJTL tasks and their subordinate elements, only 4571 were capable of being objectively quantified.

The remaining subjective or uncertain tasks leave several different aspects of combat which still remain unmeasurable. When faced with judgments about these aspects of combat while doing a causal audit trail analysis, the only alternative is to end that portion of the causal audit trail "tree" at the given subjective point.

Several critical events are analyzed in Dromerhauser and McAneny (1997). In order to demonstrate an appreciation of the methodology, the Madinah Division Withdrawal is presented in this paper.

\subsection{Critical Event: Madinah Division Withdrawal}

The critical event involves the progress of the 2/24th Mech toward the objective area. After making steady movement toward KKMC during the first day, they were impeded by a successful Iraqi air strike at 0.625 days. Damage was simulated and the resulting time of repair induced further delays. The 2/24th Mech eventually reached KKMC and engaged the Madinah Division in battle, but the effects of this delay are not clear. Madinah Division began to withdraw from contact at day 1.15 and endured a twelve hour conflict with the 2/24th Mech during their retreat. After this, Coalition forces lost contact with the Madinah, and the division was allowed to withdraw unimpeded. This sequence yields an obvious question to ask: Why was

\section{the Madinah Division allowed to withdraw unimpeded?}

In Brown's (1996) efforts to measure successful operational maneuver, he states that "some method of quantifying the first order effects of operational art must be determined." He continues by suggesting that this representation must include more than simply using the speed of any given combat system, or aggregation of systems. Instead, it must be relational, taking into account the operational maneuver of both friendly and enemy forces, as well as the descriptive characteristics of the units over time.

The need for a relational descriptive parameter led to Brown's development of a measure of performance that is called the Fractional Closure Rate, or FCR:

$$
F_{C R}(t)=\frac{\operatorname{DISTANCE}_{f, e}(t-\Delta t)-\operatorname{DISTANCE}_{f, \mathrm{e}}(t)}{\operatorname{MAX}\left[\operatorname{DISTANCE}_{f, e}(t-\Delta t)-\operatorname{DISTANCE}_{f, e}(t)\right]}, \forall f, e, t
$$

where $f=$ a specified friendly maneuver element or target

$e=$ a specified enemy maneuver element or target

$t=$ time of capture of the data.

The numerator of the FCR is a representation of the closure distance between two forces in some time interval, $\Delta t$, or more simply the approach velocity of two forces. Dividing by the maximum of the current distance between forces creates a measure which has the flexibility of demonstrating negative change in relation to the closure. Withdrawing at a certain distance has a negative FCR of the same magnitude as an advance at the same distance.

Brown further explains that "the Fractional Closure Rate is developed only as a measure of performance to be incorporated into a measure of effectiveness for maneuver. It does have some stand-alone use as a measure of effectiveness of the ability of a force to maintain a high operational tempo. This translates to a quantitative measure of initiative and agility, using depth of attack as the data element."

Brown's first application of the utility of the FCR to wargaming is an analysis of the critical event: Madinah Division Withdrawal. The progress of $2 / 24$ 's movement westward toward KKMC is exhibited in Figure 1, with curve behavior pointing to causal events which impede or facilitate that progress. The trend line, shown in black, uses every three data points to calculate a moving average, which is a characteristic representation of the overall trends in initiative and momentum.

A slow, but successful movement toward the objective area is demonstrated by the gradual rise in 


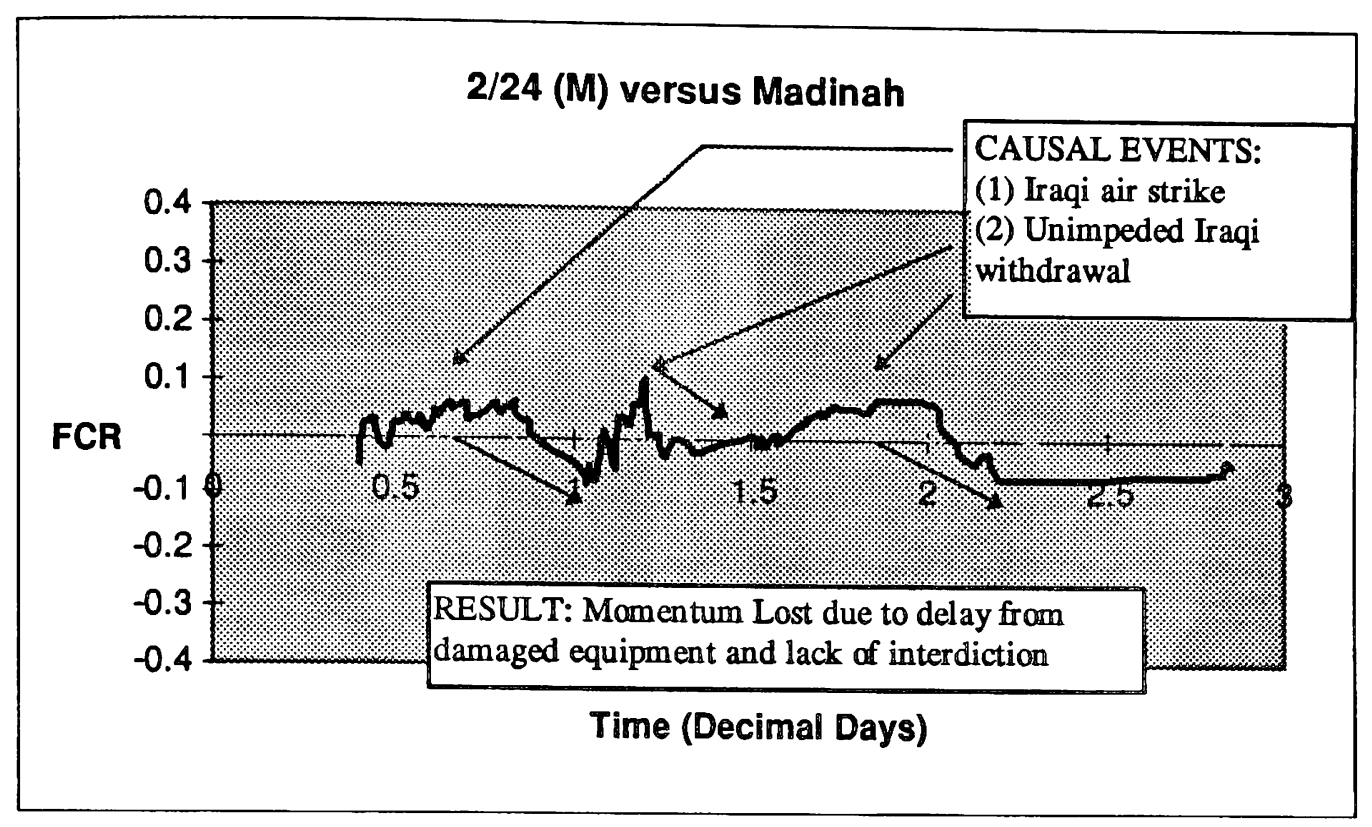

Figure 1. Effects of Interdiction on Closure

FCR from first movement until the delay caused by the Iraqi air strike at time 0.625 . Damage is simulated and the resulting time of repair induces further delays. This, in turn, produces a drop in momentum until the completion of the first day. Additionally, the initiation of the ground offensive by the Madinah Division creates movement away from the $2 / 24$ 's static location, further decreasing the closure. As the damage is repaired, the closure ratio begins to increase rapidly for approximately 0.25 days, or six hours. During this time the $2 / 24$ is able to close on KKMC and the Madinah Division, and join the battle. The more pronounced spatial acceleration, or slope of the FCR, is the result of the relational movement of the two forces moving toward the same location at this point in the battle. Madinah's movement was necessary to initiate ground combat with forces already at KKMC, thereby maintaining the operational initiative.

As Madinah begins to withdraw from contact at time 1.15 , the FCR initially drops off before stabilizing back to the gradual rise exhibited in the first few hours of the scenario. At this point in the battle Madinah begins to lose its momentum, and the unimpeded $2 / 24$ closes and joins battle at approximately time 1.5. For the next twelve hours, the Coalition force is able to create a favorable tempo, but has arrived well after the Iraqi force was able to withdraw. Though creating a favorable FCR from time 1.5 to 2.0 , it is occurring at the expense of pursuing an enemy with whom they still have not gained contact. Finally, at the beginning of the second day, Madinah continues its withdrawal uninhibited by Coalition efforts. The curve characteristics at this point are useful to a CINC in evaluating the exercise. The defeated Iraqi force is able to reduce the FCR. This translates to an inability to prevent a force from escaping the battle area. If this were intentional, then the curve is simply an affirmation that the plan was properly conducted. If not, this analysis portrays the inability of the $24^{\text {th }}$ Mech to maintain the tempo and create a favorable FCR with respect to the Iraqi forces.

The Madinah Division executes the withdrawal along with the Hammurabi Division. The movement is from the KKMC area of operations to support forces along the coast. Given the lateral movement of the Iraqi forces, a successful counterattack plan would have slowed the withdrawal.

The "decision tree" for the Madinah withdrawal is shown in Figure 2. To answer the aforementioned question of why the Madinah Division is allowed to withdraw unimpeded, the possible broad categories of causes must be delineated. Was it due to poor intelligence, $2 / 24$ 's lack of mobility, a supply shortfall, low force strength, prior tasking of $2 / 24$, or was this sequence of events nothing more than a Coalition operational tactic?

Did $2 / 24$ have any prior tasking that would somehow impede their pursuit of Madinah? In order to investigate this question, the $2 / 24$ 's mission and posture data during the period leading up to Madinah's withdrawal must be analyzed. Every unit in JTLS has both a mission and a posture. The mission is generally the last thing the unit was ordered to do. The posture describes what the unit is actually doing. This 


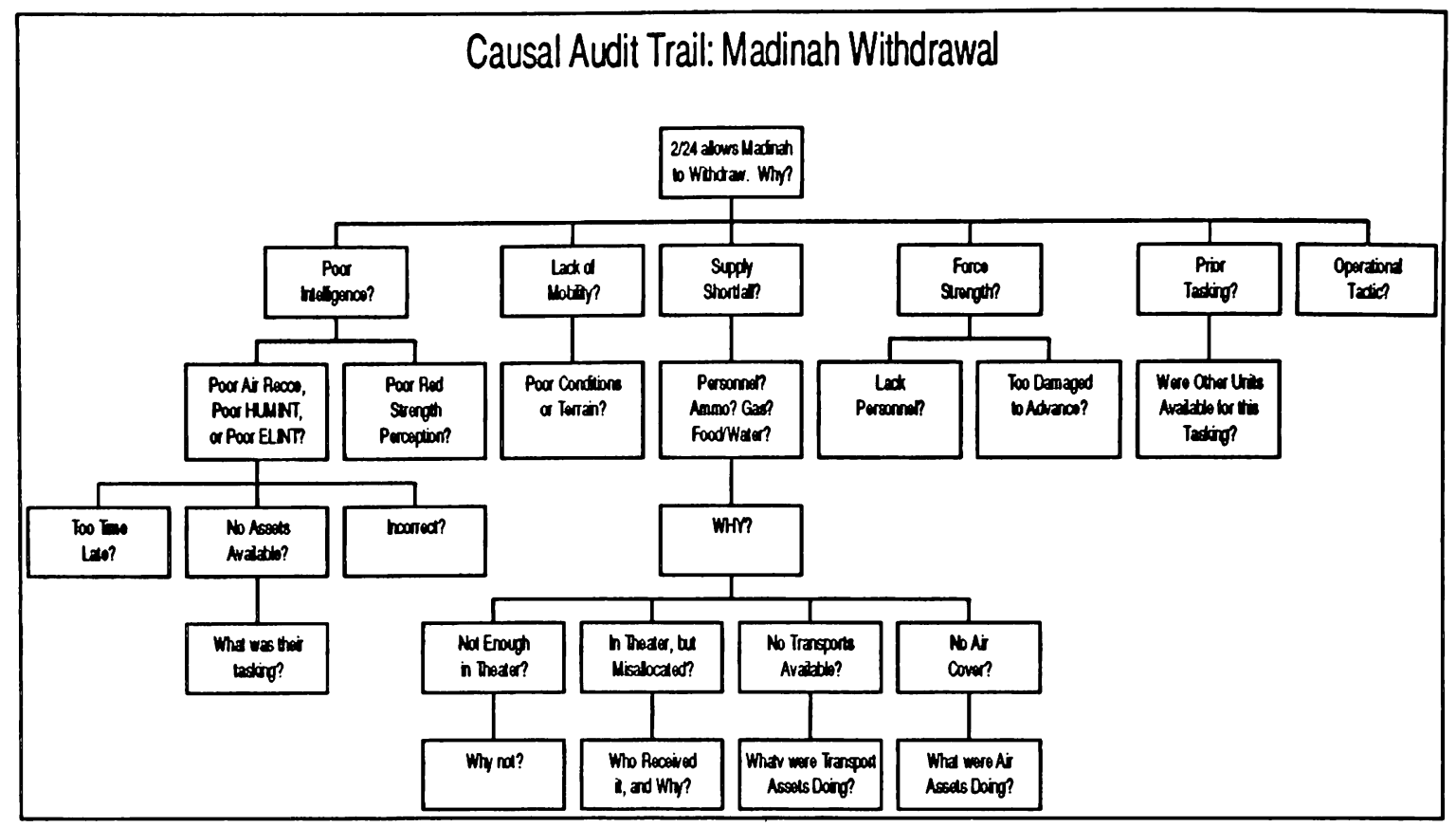

Figure 2: Event One Causal Audit Trail Decision Tree

information is available in chronological order in $2 / 24$ 's Unit Change List (Table 1), which shows that $2 / 24$ is in a MOVING posture from day 0.05526 to 0.6648 , when it then shifts to a DEFEND posture, then back to MOVING at 1.0013 . It then briefly shifts back to DEFEND at day 1.1169 until 1.5007 .

Table 1: Summary of Postures for $2 / 24$ and Madinah

\begin{tabular}{ccc}
\hline $\begin{array}{c}\text { Scenario Time } \\
\text { of Posture } \\
\text { Status Change }\end{array}$ & $\begin{array}{c}\text { 2/24's } \\
\text { Posture }\end{array}$ & $\begin{array}{c}\text { Madinah's } \\
\text { Posture }\end{array}$ \\
\hline .5526 & MOVING & ATTACK \\
.6648 & DEFEND & ATTACK \\
.9167 & DEFEND & DEFEND \\
1.0013 & MOVING & ATTACK \\
1.0016 & MOVING & WITHDRAW \\
1.1169 & DEFEND & WITHDRAW \\
1.5007 & ATTACK & WTTHDRAW \\
$\downarrow$ & $\downarrow$ & $\downarrow$ \\
\hline
\end{tabular}

These observations tell the analyst that, for some reason, 2/24's mission and posture shifted from DEFEND to MOVING almost immediately before Madinah began their withdrawal at day 1.0016. This means that $2 / 24$ had the correct mission and posture to follow Madinah, eliminating this as a possible cause for the critical event. However, at day 1.1169, 2/24 went to a DEFEND posture which stopped 2/24 from pursuing Madinah.

Was allowing Madinah to withdraw a product of poor Coalition intelligence? This branch of the decision tree would be essential in most scenarios, but due to the composition of coalition and opposing forces in this wargame, intelligence played an almost nonexistent role. No HUMINT or ELINT assets were allocated to either side, and air reconnaissance assets were not available because the Coalition forces were not allotted any air assets. This artificiality was put in place strictly for the purpose of enhancing the quantity of ground combat available for analysis. Had Coalition air forces been used, the possibility of opposing ground forces being quickly eliminated seemed very likely. The only intelligence related indicators that are available for analysis are opposing and coalition's perceptions of each others strength and location.

The 2/24 receives accurate updates of Madinah's location whenever Madinah's location changes and 2/24 is within the range of its given sensors. These sensors may be radars, intelligence sources, or in this case, eyeballs. When not within sensor range of Madinah, 2/24 perceives them to be at their last known location (Figure 3). This figure shows the distance, in nautical miles, between Madinah's actual location and where 2/24 perceives Madinah to be. As could be expected, the graph follows the timeline of the scenario: after making steady movement toward $\mathrm{KKMC}$ and staying close to Madinah during the first day, 2/24 was impeded by a 


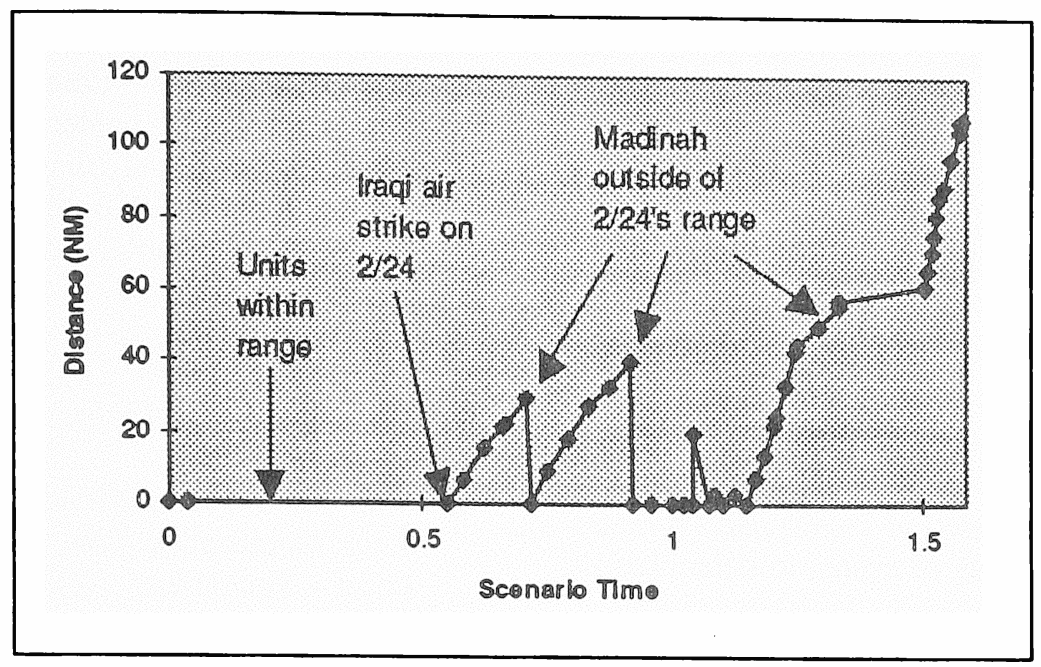

Figure 3. Difference Between Madinah's Perceived and Actual Locations

successful Iraqi air strike at 0.625 days. Damage was simulated and the resulting time of repair induced further delays. The 2/24th Mech eventually reached KKMC and engaged the Madinah Division in battle, then Madinah began to withdraw from contact at day 1.0016 and endured a twelve hour conflict with the 2/24th Mech during their retreat. After this, Coalition forces lost contact with the Madinah, and the division was allowed to withdraw unimpeded.

Of all possible branches of the causal audit trail tree, this one offers the most plausible one. The reason 2/24 did not follow Madinah in its withdrawal was almost certainly the fact that they did not know where Madinah was. Once Madinah exceeded the range of $2 / 24$ 's sensors, there were no other Coalition forces close enough to find this elusive Iraqi unit.
Similar analysis of the perception of force strength is shown in Figure 4. The 2/24 maintains an accurate picture of Madinah's force strength until they begin their withdrawal at day 1.0016 where, due to the increased range, 2/24 slightly underestimates Madinah's force strength. This can also be attributed to the lack of Coalition intelligence assets. The only Coalition asset available to assess the strength of Madinah is $2 / 24$, since it is the only unit within range to perform this task.

Had the scenario included other intelligence related data, the following elements would be essential for analysis of the critical event:

- Report all of the organic intelligence data regarding Madinah forwarded during the entire scenario, and compare with the actual times of events to determine the age of the information.

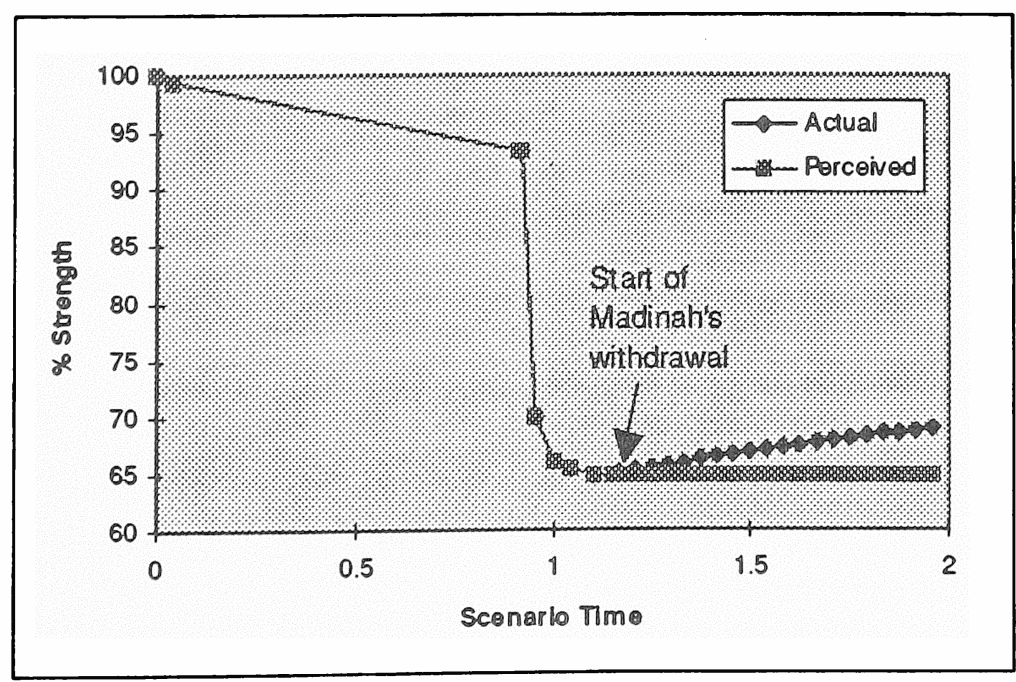

Figure 4. Madinah's Perceived and Actual Strength vs. Time 
- Report which Coalition air recce assets were available and their tasking during the period leading up to Madinah's withdrawal.

- Report all air recce intelligence data forwarded during the given period of inquiry, and compare with the actual times of events to determine how time late they are.

- Report all Coalition ELINT and HUMINT assets available during the given period of inquiry and their tasking during the same period.

- Report all ELINT and HUMINT data forwarded during the given period of inquiry, and compare with the actual times of events to determine its relevance.

Searching for the most likely cause of this critical event reveals that the "poor intelligence" branch of the causal audit trail tree is the likely cause. Ignoring any potential subjective decisions that may have affected this scenario, the Coalition forces most glaring Achilles' heel was their inability to track the enemy. This can be attributed almost entirely to their complete lack of deployed intelligence assets.

\section{REFERENCES}

Brown, K. 1996. Evaluating Operational Maneuver in a Computer Aided Exercise. Master's Thesis, Operations Research Department, Naval Postgraduate School, Monterey, California.

Coleman Research Corporation Final Report, January 1997. Training Proficiency Evaluation Support Module. Huntsville, Alabama.

Combs, R. 1995. A Methodology for Evaluating Execution of Universal Joint Tasks Within the Context of a Computer Aided Exercise. Master's Thesis, Operations Research Department, Naval Postgraduate School, Monterey, California.

Cwick, M. 1996. Analyzing Amphibious Logistics Capabilities in the Joint Theater Level Simulation (JTLS). Master's Thesis, Operations Research Department, Naval Postgraduate School, Monterey, California.

Gordon, K. 1996. A Methodology for Evaluating Operational Firepower During a Computer Aided Exercise. Master's Thesis, Operations Research Department, Naval Postgraduate School, Monterey, California.

Headquarters, Joint Warfighting Center. April 1995. D-J-0013-K, Joint Theater Level Simulation Analyst Guide, Fort Monroe, Virginia.

Joint Chiefs of Staff. 1996. Universal Joint Task List. CJCSM 3500.04, Version 2.1.
McAneny, M.C. and Dromerhauser, R.J. 1997. A Methodology for Comprehensive Quantitative Evaluation of a Computer Aided Exercise Using the Joint Theater Level Simulation (JTLS). Master's Thesis, Operations Research Department, Naval Postgraduate School, Monterey, California.

Mustin, J. 1996. Evaluating Carrier Battlegroup AntiAir Warfare Capability in a Computer Aided Exercise. Master's Thesis, Operations Research Department, Naval Postgraduate School, Monterey, California.

Sullivan, M. 1996. A Methodology for Evaluating a Joint Mobilization Plan Using the Joint Theater Level Simulation (JTLS). Master's Thesis, Operations Research Department, Naval Postgraduate School, Monterey, California.

Thurman, J. 1996. A Methodology for Evaluating Force Protection During a Computer Aided Exercise. Master's Thesis, Operations Research Department, Naval Postgraduate School, Monterey, California.

Towery, C. 1995. A Methodology for Evaluating Intelligence Functions During a Computer Exercise. Master's Thesis, Operations Research Department, Naval Postgraduate School, Monterey, California.

\section{AUTHOR BIOGRAPHIES}

SAM H. PARRY is a Professor of Operations Research at the Naval Postgraduate School. He received his Ph.D. degree in Systems Engineering from The Ohio State University. His research interests are in AirLandSea Combat modeling and is currently Principal Investigator for two research projects sponsored by the Office of the Secretary of Defense and the Joint Chiefs of Staff. He is a member of the Military Operations Research Society.

LT MICHAEL C. MCANENY, JR. is a Master's student in the Operations Research Department at the Naval Postgraduate School. He received his B.S. from the U.S. Naval Academy in 1991. He is a Naval Officer with Surface Warfare experience serving on USS YORKTOWN as Navigator, Ordnance Officer, and Auxiliaries Officer.

LT RICHARD J. DROMERHAUSER, JR. is a Master's student in the Operations Research Department at the Naval Postgraduate School. He received his B.S. from the U.S. Naval Academy in 1990. He is a Naval Officer having served in Engineering billets on Arleigh Burke class destroyers and Mine Countermeasures ships. 